\title{
COINCIDENCE POINT THEOREMS FOR MULTI-VALUED AND SINGLE-VALUED MAPPINGS IN MENGER PM-SPACES
}

\author{
H. K. PATHAK, Y. J. CHO, S. S. CHANG AND S. M. KANG
}

\begin{abstract}
In this paper, coincidence point theorems for compatible mappings of multi-valued and single-valued mappings in Menger PM-spaces are obtained. The presented results in this paper generalize and improve the corresponding results of S. S. Chang et al., O. Hadžić and others.
\end{abstract}

Throughout this paper, let $R=(-\infty, \infty), R^{+}=[0, \infty), Z^{+}$be the set of all positive integers and $\mathcal{D}$ be the set of all distribution finctions on $R$.

If $(X, \mathcal{F}, T)$ is a Menger probabilistic metric space (shortly, a Menger PM-space) with the continuous $t$-norm $T$, then $(X, \mathcal{F}, T)$ is a Hausdorff space [6] in the topology $\tau$ induced by the faimily

$$
\left\{U_{x}(\epsilon, \lambda): x \in X, \epsilon>0, \lambda \in(0,1)\right\}
$$

of neighborhoods $U_{x}(\epsilon, \lambda)$, where

$$
U_{x}(\epsilon, \lambda)=\left\{y \in X: F_{x, y}(\epsilon)>1-\lambda\right\}
$$

Refer to [1], [2] and [6]-[8] for other details on PM-spaces.

Definition 1. Let $(X, \mathcal{F}, T)$ be a Menger PM-space with the continuous $t$-norm $T$ and $A$ be a nonempty subset of $X . D_{A}(t)=\sup _{s<t} \inf _{x, y \in A} F_{x, y}(s)$ is called the probabilistic diameter of $A$. Specifically, the set $A$ is said to be probabilistically bounded if

$$
\lim _{t \rightarrow \infty} D_{A}(t)=1
$$

Received March 16, 1994; revised June 7, 1994.

1991 Mathematics Subject Classification. 54H25.

Key words and phrases. Menger PM-spaces, probabilistic distances, $t$-norm of $h$-type, weakly compatible mappings and common fixed points.

This paper was partially supported by U.G.C., 1994, New Delhi, India, and the Young-Joong Hur Research Foundation of Gyeongsang National University, 1994. 
From [2], we know that

(1) if $A$ is a probabilistically bounded subset of $X$, then $D_{A}(t)$ is a distribution function,

(2) if $A$ and $B$ are probabilistically bounded subsets of $X$, then $A \cup B$ is also a probabilistically bounded set.

Let $(X, \mathcal{F}, T)$ be a Menger PM-space with the continuous $t$-norm $T$ and $C B(X)$ be the family of nonempty probabilistically bounded and closed subset of $X$. We define a mapping $\widetilde{\mathcal{F}}: C B(X) \times C B(X) \rightarrow \mathcal{D}$ as follows (we denote $\widetilde{\mathcal{F}}\left(A, B\right.$ ) by $\widetilde{F}_{A, B}$ and the value $\widetilde{F}(A, B)$ at $t \in R$ by $\left.\widetilde{F}_{A, B}(t)\right)$ :

$$
\widetilde{F}_{A, B}(t)=\sup _{s<t} T\left(\inf _{x \in A} \sup _{y \in B} F_{x, y}(s), \inf _{y \in B} \sup _{x \in A} F_{x, y}(s)\right), \quad A, B \in C B(X) .
$$

The mapping $\widetilde{\mathcal{F}}$ is called the Menger-Hausdorff metric induced by $\mathcal{F}$.

Proposition 1. [2] $(C B(X), \widetilde{F}, T)$ be a Menger PM-space, i.e., $\widetilde{F}$ is a mapping satisfying the following conditions:

(1) $\widetilde{F}_{A, B}(t)=1$ for all $t>0$ if and only if $A=B$,

(2) $\widetilde{F}_{A, B}(0)=0$,

(3) $\widetilde{F}_{A, B}(t)=\widetilde{F}_{B, A}(t)$,

(4) $\widetilde{F}_{A, B}\left(t_{1}+t_{2}\right) \geq T\left(\widetilde{F}_{A, C}\left(t_{1}\right), \widetilde{F}_{C, B}\left(t_{2}\right)\right)$ for all $A, B \in C B(X)$ and $t_{1}, t_{2}>0$.

Definition 2. Let $A \in C B(X)$ and $x \in X$. The probabilistic distance between $x$ and $A$ is the function defined by

$$
F_{x, A}(t)=\sup _{s<t} \sup _{y \in A} F_{x, y}(s), \quad t>0
$$

Proposition 2. [2] Let $A, B \in C B(X)$ and $x, y \in X$. Then we have the following:

(1) $F_{x, A}(t)=1$ for all $t>0$ if and only if $x \in A$,

(2) $F_{x, A}\left(t_{1}+t_{2}\right) \geq T\left(F_{x, y}\left(t_{1}\right), F_{y, A}\left(t_{2}\right)\right)$ for all $t_{1}, t_{2}>0$,

(3) $F_{x, B}(t) \geq \widetilde{F}_{A, B}(t)$ for all $t>0$.

Remark 1. If we define $F_{x, A}(t)=\sup _{y \in A} F_{x, y}(t)$, then, since we have

$$
\sup _{s<t} \sup _{y \in A} F_{x, y}(s)=\sup _{y \in A} F_{x, y}(t)
$$

$F_{x, A}(t)$ is a left-continuous at $t$ and the properties (1), (2), (3) of Proposition 2 are also satisfied.

Definition 3. Let $\phi: R^{+} \rightarrow R^{+}$be a strictly increasing function such that $\phi(0)=0$ and $\lim _{t \rightarrow \infty} \phi(t)=+\infty$. Define a function $\psi: R^{+} \rightarrow R^{+}$by

$$
\psi(t)= \begin{cases}0 & t=0 \\ \inf \{s>0: \phi(s)>t\}, & t>0\end{cases}
$$


It is easy to prove that $\psi: R^{+} \rightarrow R^{+}$is a continuous and nondecreasing function.

Definition 4. We say that a function $\phi: R^{+} \rightarrow R^{+}$satisfies the condition $(\Phi)$ if it is a strictly increasing and left-continuous function such that $\phi(0)=0, \lim _{t \rightarrow+\infty} \phi(t)=$ $+\infty$ and $\sum_{n=0}^{\infty} \phi^{n}(t)<+\infty$ for all $t>0$, where $\phi^{n}(t)$ is the $n$-th iteration of $\phi(t)$.

Lemma 3. [3] Let $\phi: R^{+} \rightarrow R^{+}$satisfy the condition ( $\Phi$ ) and let $\psi$ be defined by (A). Then we have the following:

(1) $\phi(t)<t$ for all $t>0$,

(2) $\phi(\psi(t)) \leq t$ and $\psi(\phi(t))=t$ for all $t \geq 0$,

(3) $\psi(t) \geq t$ for all $t \geq 0$,

(4) $\lim _{n \rightarrow \infty} \psi^{n}(t)=+\infty$ for all $t>0$.

Definition 5. A $t$-norm $T$ is said to be of $h$-type if the family $\left\{T^{m}(t)\right\}_{m=1}^{\infty}$ of the functions $T^{m}(t)=T\left(t, T^{m-1}(t)\right), m=1,2, \ldots, T^{0}(t)=t, t \in[0,1]$, is equicontinuous at 1.

Lemma 4. [3] Let $(X, \mathcal{F}, T)$ be a Menger PM-space, where $T$ is a t-norm of $h$-type. If a sequence $\left\{x_{n}\right\}$ in $X$ satisfies the following condition:

For any $n \in Z^{+}$and $t>0$,

$$
F_{x_{n}, x_{n+1}}(t) \geq F_{x_{0}, x_{1}}\left(\psi^{n}(t)\right)
$$

where $\phi$ is a function satisfying the condition $(\Phi)$ and $\psi$ is defined by $(\mathrm{A})$, then the sequence $\left\{x_{n}\right\}$ is a Cauchy sequence in $X$.

Now we introduce the concepts of weakly compatible mappings for multi-valued and single-valued mappings in the setting of PM-spaces, which is motivated by the concept of compatible mappings for single-valued mappings in metric spaces [5].

Definition 6. Let $f$ be a mapping from $X$ into itself and $S$ be a multi-valued mappings $X$ into $C B(X)$.

(1) The mappings $f$ and $S$ are said to be commuting if $f S x \in C B(X)$ and $f S x=$ $S f x$ for all $x \in X$,

(2) The mappings $f$ and $S$ are said to be weakly compatible if $f S x=C B(X)$ and

$$
\lim _{n \rightarrow \infty} \widetilde{F}_{f S x_{n-1}, S f x_{n-1}}(t)=1, \quad t>0,
$$

whenever $\left\{x_{n}\right\}$ is a sequence in $X$ such that

$$
\lim _{n \rightarrow \infty} F_{f x_{n}, S x_{n-1}}(t)=1, \quad t>0 .
$$

Remark 2. From Definition 6, any commuting mappings are weakly compatible but the converse is not true (cf. [5]). 
Now we are in a position to give our main results:

Theorem 5. Let $(X, \mathcal{F}, T)$ be a complete Menger PM-space with the continuous t-norm $T$ of h-type. Let $f$ be a continuous self-mapping of $X$ and $S$ be a continuous multi-valued mapping from $X$ into $C B(X)$ satisfying the following conditions:

(C) $S(X) \subset f(X)$,

(D) For any $x, y \in X$ and $u \in S x$, there exists a point $v \in S y$ such that

$$
F_{u, v}(\phi(t)) \geq \min \left\{F_{f x, f y}(t), F_{f x, S x}(t), F_{f y, S y}(t)\right\}
$$

for all $t>0$, where the function $\phi$ satisfies the condition $(\Phi)$.

If $f$ and $S$ are weakly compatible, then there exists a point $z \in X$ such that $f z \in S z$, that is, the point $z$ is a coincidence point of $f$ and $S$.

Proof. First, we shall show that we can find a sequence $\left\{x_{n}\right\}_{n \in Z^{+}}$in $X$ such that

$$
F_{f x_{n}, f x_{n+1}}(t) \geq F_{f x_{0}, f x_{1}}\left(\psi^{n}(t)\right)
$$

for all $t>0$ and $n \in Z^{+}$.

From (C), for arbitrary $x_{0} \in X$, there exists a point $x_{1} \in X$ such that $f x_{1} \in S x_{0}$. By Lemma 3 and (D), there exists a point $x_{2} \in X$ such that $f x_{2} \in S x_{1}$ and

$$
\begin{aligned}
F_{f x_{1}, f x_{2}}(t) & \geq F_{f x_{1}, f x_{2}}(\phi(\psi(t))) \\
& \geq \min \left\{F_{f x_{0}, f x_{1}}(\psi(t)), F_{f x_{0}, S x_{0}}(\psi(t)), F_{f x_{1}, S x_{1}}(\psi(t))\right\} \\
& \geq \min \left\{F_{f x_{0}, f x_{1}}(\psi(t)), F_{f x_{1}, f x_{2}}(\psi(t))\right\}
\end{aligned}
$$

for all $t>0$, where $\psi(t)$ is defined by $(\mathrm{A})$. Using (E) repeatedly, we have

$$
\begin{aligned}
F_{f x_{1}, f x_{2}}(t) & \geq \min \left\{F_{f x_{0}, f x_{1}}(\psi(t)), F_{f x_{0}, f x_{1}}\left(\psi^{2}(t)\right), F_{f x_{1}, f x_{2}}\left(\psi^{2}(t)\right)\right\} \\
& =\min \left\{F_{f x_{0}, f x_{1}}(\psi(t)), F_{f x_{1}, f x_{2}}\left(\psi^{2}(t)\right)\right\} \\
& \geq \cdots \\
& \geq \min \left\{F_{f x_{0}, f x_{1}}(\psi(t)), F_{f x_{1}, f x_{2}}\left(\psi^{n}(t)\right)\right\} .
\end{aligned}
$$

Letting $n \rightarrow \infty$ in $(F)$, we have

$$
F_{f x_{1}, f x_{2}}(t) \geq F_{f x_{0}, f x_{1}}(\psi(t)), \quad t>0 .
$$

By repeating the above procedure, we can define a sequence $\left\{x_{n}\right\}$ in $X$ such that $f x_{n+1} \in$ $S x_{n}$ and

$$
F_{f x_{n}, f x_{n+1}}(t) \geq F_{f x_{n-1}, f x_{n}}(\psi(t)), \quad t>0 .
$$

Thus, for any $n \in Z^{+}$and $t>0$, we have

$$
F_{f x_{n}, f x_{n+1}}(t) \geq F_{f x_{n-1}, f x_{n}}(\psi(t)) \geq \cdots \geq F_{f x_{0}, f x_{1}}\left(\psi^{n}(t)\right)
$$


and so, by Lemma $4,\left\{f x_{n}\right\}$ is a Cauchy sequence in $X$. Since $(X, \mathcal{F}, T)$ is complete, we can assume that $\lim _{n \rightarrow \infty} f x_{n}=z \in X$. Since $f$ and $S$ are weakly compatible and the sequence $\left\{x_{n}\right\}$ in $X$ is such that $f x_{n} \in S x_{n-1}$, by Proposition 2, we have

$$
\lim _{n \rightarrow \infty} F_{f x_{n}, S x_{n-1}}(t)=1, \quad t>0
$$

and so

$$
\lim _{n \rightarrow \infty} \widetilde{F}_{f S x_{n-1}, S f x_{n-1}}(t)=1, \quad t>0 .
$$

On the other hand, from (3) of Proposition 2, it follows that

$$
F_{f f x_{n}, S f_{n-1}}(t) \geq \widetilde{F}_{f S x_{n-1}, S f x_{n-1}}(t), \quad t>0 .
$$

Letting $n \rightarrow \infty$ in $(H)$, we have

$$
1=\lim _{n \rightarrow \infty} F_{f f x_{n}, S f_{n-1}}(t)=F_{f z, S z}(t)
$$

and so, by Proposition 2, $f z \in S z$, that is, $z$ is a coincidence point of $f$ and $S$. This completes the proof.

In Theorem 5 , by strengthening the weak compatibility of $f$ and $S$ by the commutitivity of $f$ and $S$, we have the following:

Theorem 6. Let $(X, \mathcal{F}, T)$ be a complete Menger PM-space with the continuous t-norm $T$ of $h$-type. Let $f$ and $S$ be as in Theorem 5 satisfying the conditions (C) and (D). If $f$ and $S$ commute, then there exists a point $z \in X$ such that $f z \in S z$.

Proof. Following the same argument as in Theorem 5, we can assume that $f x_{n} \rightarrow$ $z \in X$.

Next, we prove that $z$ is a coincidence point of $f$ and $S$. In fact, for any $t>0$ and $\epsilon \in(0, t)$, from (G) and the commutativity of $f$ and $S$, we have

$$
\begin{aligned}
& F_{f f x_{n+1}, S z}(t-\epsilon) \\
\geq & F_{f f x_{n+1}, S z}(\phi(\psi(t-\epsilon))) \\
= & \sup _{f y \in S z} F_{f f x_{n+1}, f y}(\phi(\psi(t-\epsilon))) \\
\geq & \min \left\{F_{f f x_{n}, f z}(\psi(t-\epsilon)), F_{f f x_{n}, f f x_{n+1}}(\psi(t-\epsilon)), F_{f z, S z}(\psi(t-\epsilon))\right\} \\
\geq & \min \left\{F_{f f x_{n}, f z}(\psi(t-\epsilon)), F_{f f x_{0}, f f x_{1}}\left(\psi^{n+1}(t-\epsilon)\right), F_{f z, S z}(\psi(t-\epsilon))\right\} .
\end{aligned}
$$

If $F_{f z, S z}(\psi(t-\epsilon))=1$, then we have

$$
F_{f f x_{n+1}, S z}(t-\epsilon) \geq \min \left\{F_{f f x_{n}, f z}(\psi(t-\epsilon)), F_{f f x_{0}, f f x_{1}}\left(\psi^{n+1}(t-\epsilon)\right)\right\} .
$$


Letting $n \rightarrow \infty$ in $(J)$, we have $F_{f z, S z_{0}}(t-\epsilon) \geq 1$. Since $\epsilon \in(0, t)$ is arbitrary, we have $F_{f z, S z}(t)=1$ for all $t>0$, that is, $f z \in S z$.

If $F_{f z, S z}(\psi(t-\epsilon))<1$, then, letting $n \rightarrow \infty$ in (I), we have

$$
F_{f z, S z}(t-\epsilon) \geq F_{f z, S z}(\psi(t))
$$

By using (2) of Proposition 2, we have

$$
F_{f z, S z}(t) \geq F_{f z, S z}(t-\epsilon) .
$$

Thus, as $\epsilon \rightarrow t$, from the continuity of $\psi$ and the left-continuity of $F_{f z, S z}$, it follows that

$$
F_{f z, S z}(t) \geq F_{f z, S z}(\psi(t))
$$

Taking this procedure repeatedly, we obtain

$$
F_{f z, S z}(t) \geq F_{f z, S z}(\psi(t)) \geq \cdots \geq F_{f z, S z}\left(\psi^{n}(t)\right)
$$

Therefore, as $n \rightarrow \infty, F_{f z, S z}(t)=1$ for all $t>0$, that is, $f z \in S z$. This completes the proof.

Taking $f=$ the identity mapping on $X$ in Theorem 6, we obtain the following:

Corollary 7. [3] Let $(X, \mathcal{F}, T)$ be a complete Menger PM-space with the continuous t-norm $T$ of h-type. Let $S$ be a multi-valued mappings from $X$ into $C B(X)$ satisfy the condition:

(M) For any $x, y \in X$ and $u \in S x$, there exists a point $v \in S y$ such that

$$
F_{u, v}(\phi(t)) \geq \min \left\{F_{x, y}(t), F_{x, S x}(t), F_{y, S y}(t)\right\}
$$

for all $t>0$, where the function $\phi$ satisfies the condition $(\Phi)$. Then $S$ has a fixed point in $X$, that is, there exists a point $z \in X$ such that $z \in S z$.

Taking $\phi(t)=k t, 0<k<1$, in Corollary 7 , we have the following:

Corollary 8. Let $(X, \mathcal{F}, T)$ be a complete Menger PM-space with the continuous $t$-norm $T$ of h-type. Let $S$ be a multi-valued mapping from $X$ into $C B(X)$ satisfying the following condition:

(N) For any $x, y \in X$ and $u \in S x$, there exists a point $v \in S y$ such that

$$
F_{u, v}(k t) \geq \min \left\{F_{x, y}(t), F_{x, S x}(t), F_{y, S y}(t)\right\}
$$

for all $t>0$, where $k \in(0,1)$ is a constant. Then $S$ has a fixed point in $X$.

Corollary 8 is a generalization of the following result of O. Hadžić ([4]): 
Corollary 9. Let $(X, \mathcal{F}, T)$ be a complete Menger PM-space with continuous t-norm $T, M$ be a nonempty probabilistically bounded subset of $X$. Let $S$ be a multi-valued mapping from $M$ into $C(M)$, the family of nonempty compact subsets of $X$, satisfying the following condition:

(O) For any $x, y \in M$ and $u \in S x$, there exists a point $v \in S y$ such that

$$
F_{u, v}(k t) \geq F_{x, y}(t)
$$

for all $t>0$, where $k \in(0,1)$ is a constant. Then $S$ has a fixed point in $M$.

\section{References}

[1] S. S. Chang, Fixed point theory with applications, Chongqing Publishing House, Chongqing, 1984.

[2] S. S. Chang, "On the theory of probabilistic metric spaces with applications," Acta Math. Sinica, New Series, 1(4), 366-377, 1985.

[3] S. S. Chang, Y. J. Cho and S. M. Kang, Common fixed point theorems for multi-valued mappings in Menger PM-spaces, to appear in Math. Japonica.

[4] O. Hadžić, "Fixed point theorems for multi-valued mappings in some classes of fuzzy metric spaces," Fuzzy Sets and Systems, 29, 115-125, 1989.

[5] G. Jungck, "Compatible mappings and common fixed points," Internat. J. Math. and Math. Sci., $29,115-125,1989$.

[6] B. Schweizer and A. Sklar, "Statistical metric spaces," Pacific J. Math., 10, 313-334, 1960.

[7] B. Schweizer and A. Sklar, Probabilistic Metric Spaces, North-Holland, New York, 1983.

[8] B. Schweizer, A. Sklar and E. Thorp, "The metrization of statistical metric spaces," Pacific J. Math., 10, 673-675, 1960.

Department of Mathematics, Kalayan Mahavidyalaya, Bhilai Nagar (M.P.) 490006, India. Department of Mathematics, Gyeongsang National University, Chinju 660-701, Korea.

Department of Mathematics, Sichuan University, Chengdu, Sichuan 610064, P. R. China.

Department of Mathematics, Gyeongsang National University, Chinju 660-701, Korea. 